\title{
Precise determination of the strong coupling in lepton collisions
}

\section{Zoltán Trócsányi*†}

Institute for Theoretical Physics, ELTE Eötvös Loránd University,

Pázmány Péter sétány 1/A, H-1117 Budapest, Hungary

and MTA-DE Particle Physics Research Group, H-4010 Debrecen, PO Box 105, Hungary

E-mail: Zoltan.Trocsanyi@cern.ch

\begin{abstract}
We discuss the status of determination of the strong coupling with special attention to using event shape observables based on data collected at the Large Electron Positron collider and theoretical predictions at highest accuracy available at present. We argue that such extractions can be competitive with lattice determination if the observables are selected carefully such that both higher order perturbative as well as non-perturbative contributions are suppressed.
\end{abstract}

International Conference on Precision Physics and Fundamental Physical Constants - FFK2019 9-14 June, 2019

Tihany, Hungary

* Speaker.

${ }^{\dagger}$ Work supported by grant K 125105 of the National Research, Development and Innovation Fund in Hungary. 
The strong sector of the standard model of particle interactions and its coupling $\alpha_{\mathrm{s}}\left(M_{Z}\right)$ is known much less precisely than the electroweak part. The current world average of its value is $\alpha_{\mathrm{s}}\left(M_{Z}\right)=0.1181 \pm 0.0011$ [1], which is dominated by determination on the lattice [2]. The values measured using experimental data span a much larger range, over $4 \%$ [3], which suggests that the extractions of $\alpha_{\mathrm{s}}\left(M_{Z}\right)$ are unlikely to reach an uncertainty below $1 \%$. Yet it is somewhat disturbing that the average of $\alpha_{\mathrm{s}}\left(M_{Z}\right)$ extractions from collider data is about one standard deviation smaller than the world average, calling for a better understanding of how to determine the strong coupling at colliders.

The extractions of $\alpha_{\mathrm{s}}\left(M_{Z}\right)$ from collider data are typically shown with three or four types of uncertainties: (i) the experimental one (exp.) that includes the statistical and experimental systematic uncertainties, (ii) the hadronization (had.) uncertainty, (iii) that due to the truncation of the perturbation series (ren.), and (iv) the resummation (res.) uncertainty if it is applicable. Presently, the experimental uncertainties are usually smaller than the other three. Hence, a more precise determination of the strong coupling requires better understanding and control of the last three types, especially the typically largest one (ren.) and the second largest one (had.).

The uncertainty due to the truncation of the perturbation series at fixed order is usually estimated by the variation of the perturbative scales-the renormalization scale and in the case of hadron collisions also the factorization scale-within a range around a default scale. Both the range and the default scale are to some extent arbitrary, and their choices can only be justified if even higher order corrections are computed. In lepton collisions the usual default scale is the total centre-of-mass energy of the collision and the range is chosen [1/2,2] times the default one.

As strong interaction processes play a role only in the final state of lepton collisions, during the operation of the Large Electron Positron Collider (LEP) such an experiment was considered an ideal place to measure $\alpha_{\mathrm{s}}\left(M_{Z}\right)$. Event shape variables constructed from geometric properties of hadronic final states (i) are sensitive to $\alpha_{\mathrm{s}}\left(M_{Z}\right)$, (ii) were measured extensively at LEP and assuming the validity of local parton-hadron duality (iii) can almost be computed from first principles. Hence they provide the tools for extractions of $\alpha_{\mathrm{s}}\left(M_{Z}\right)$ from hadronic data. According to our current view of high energy elementary particle collisions, the hard process produces several initial partons of high virtuality, each of which being a seed for a shower of partons, produced by a sequence of collinear parton splittings. These processes are described by the quantum field theory of QCD and the precision of the theoretical precision depends only on our ability to perform the complex computations in perturbation theory.

During the last 15 years there has been an impressive improvement in the computations of radiative corrections to the distributions of event shapes. On the one hand the next-to-next-toleading order (NNLO) corrections have been computed for three-jet like observables [4, 5, 6]. On the other hand resummation of large logarithms, which are characteristic features of collinear and soft parton splittings, have been performed to all orders at the next-to-next-to-leading logarithmic (NNLL or $\mathrm{N}^{2} \mathrm{LL}$ )) and in some cases even at $\mathrm{N}^{3} \mathrm{LL}$ accuracy $[7,8,9]$. Both lead to a significant decrease of the dependence on the renormalization scale.

The detectors however detect hadrons and we do not have a theory of the process of hadronization in which partons turn into hadrons. Hence, in spite of the impressive improvement in the precision of perturbative computations, the predictions still differ significantly from the measurements for most of the event shape distributions. An example is shown in Fig. 1 (left) where the distribu- 

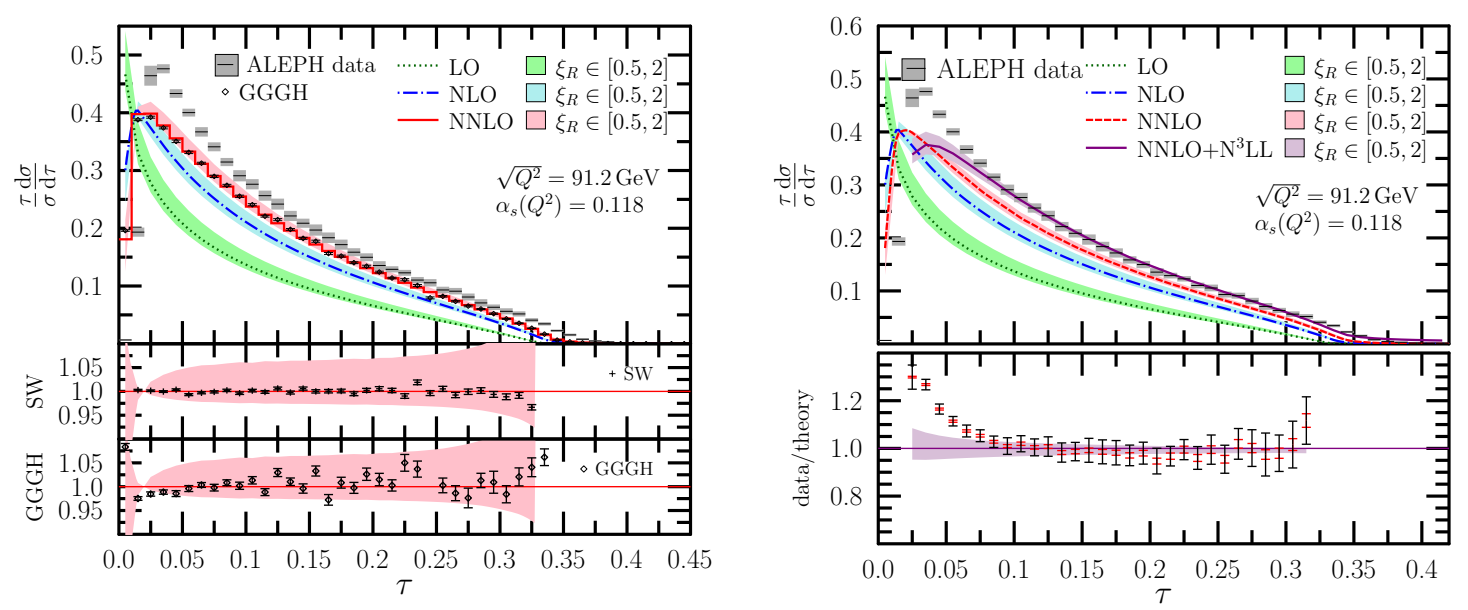

Figure 1: Distributions of thrust in electron-positron annihilation normalized to the total hadornic cross section $\sigma$. Left: predictions at first three orders in perturbation theory and also matched NNLO and $\mathrm{N}^{3} \mathrm{LL}$ predictions obtained with the world average for the strong coupling. Right: distribution of matched NNLO and $\mathrm{N}^{3}$ LL predictions supplemented with analytic model for power corrections fitted to ALEPH data. The bands represent the variation of the renormalization scale $\mu_{R}=\xi_{R} \sqrt{Q^{2}}$ around the default one $\left(\xi_{R}=1\right)$ where $\sqrt{Q^{2}}$ is the total centre-of-mass energy. The range $\xi_{R} \in[0.5,2]$ is the accepted measure of the uncertainty in the perturbative predictions at fixed order in perturbation theory. The lower panels show the ratio of the (updated but unpublished) predictions of Ref. [5] (SW) and the EERAD3 code [15] (GGGH) to CoLoRFuINNLO.

tion of thrust $T[10,11](\tau=1-T)$ can be seem at LO, NLO and NNLO accuracy, as given by the perturbative expansion for the normalized cross section, ${ }^{1}$

$$
\frac{\tau}{\sigma} \frac{\mathrm{d} \sigma}{\mathrm{d} \tau}=\left(\frac{\alpha_{\mathrm{s}}}{2 \pi}\right) A(\tau)+\left(\frac{\alpha_{\mathrm{s}}}{2 \pi}\right)^{2} B(\tau)+\left(\frac{\alpha_{\mathrm{s}}}{2 \pi}\right)^{3} C(\tau) .
$$

We see that even the most precise fixed-order prediction (NNLO) falls much below the data, especially in the region of small $\tau$ where $\operatorname{logarithms} L=-\ln \tau$ become large. The reason is easy to understand from the analytic structure of perturbative predictions:

$$
\begin{aligned}
& A(\tau)=A_{1} L+A_{0}, \\
& B(\tau)=B_{3} L^{3}+B_{2} L^{2}+B_{1} L+B_{0}, \\
& C(\tau)=C_{5} L^{5}+C_{4} L^{4}+C_{3} L^{3}+C_{2} L^{2}+C_{1} L+C_{0}
\end{aligned}
$$

where the dependence of the coefficients on $\tau$ is suppressed. The logarithmic contributions can be of $\mathrm{O}(1)$ at each order in $\alpha_{\mathrm{s}}$, hence have to be resummed to all orders in order to obtain a reliable prediction for small values of $\tau$. As shown in Fig. 1 (right), combining the NNLO and $N^{3} L L$ predictions, using R-matching [16] to avoid double counting of logarithmic terms appearing both in the fixed-order and resummed predictions, improves the agreement between the prediction and data for the thrust distribution significantly, but not sufficiently. There is still a large difference

\footnotetext{
${ }^{1}$ The $A, B$ and $C$ coefficients were computed using the MCCSM program [12] that implements the CoLoRFuINNLO subtraction method $[13,14]$.
} 

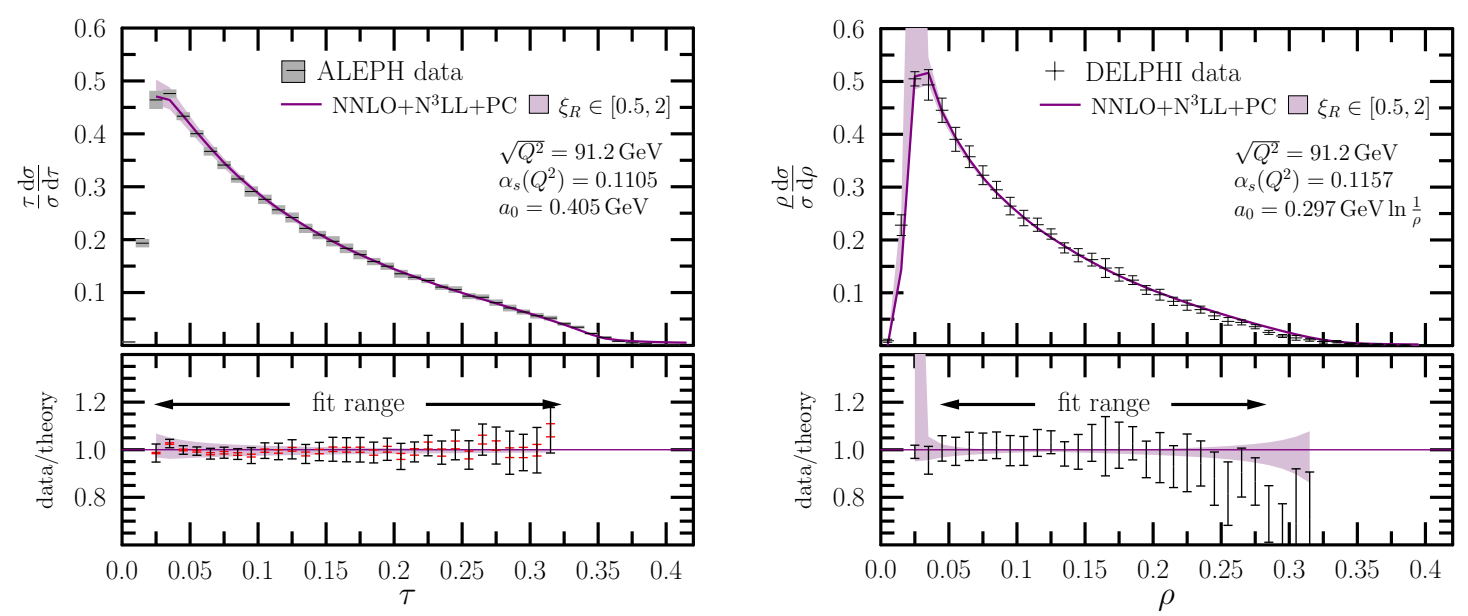

Figure 2: Distributions of thrust ( $\tau$, left) and heavy jet mass ( $\rho$, right) in electron-positron annihilation, normalized to the total hadronic cross section $\sigma$ and using matched NNLO and $\mathrm{N}^{3}$ LL predictions supplemented with power corrections for hadronization fitted to Delphi data. The bands represent the variation of the renormalization scale around the default one as in Fig. 1.

between the predictions and data in the peak region where most of the data fall. We believe that these differences are due to the effect of the hadronization.

We have two options to estimate the effects of hadronization. One is the use of an analytic model for the non-perturbative corrections (power corrections, PC) $[17,7,18]$, which essentially amounts to a shift of the differential distribution. The second one is to use modern Monte Carlo tools to estimate the effect by simulating the final states both at the parton and at the hadron level and use the ratio of the two as a multiplicative correction factor (fitted with a smooth function) $[19,20]$. Both were applied in the past in extractions of $\alpha_{\mathrm{s}}\left(M_{Z}\right)$ from collider data. The fits for the strong coupling and the non-perturbative parameter simultaneously, based on using NLO+NLL accurate predictions together with analytic model for the power corrections, did not show universality [21]. This feature has not improved with the inclusion of higher order radiative corrections [22]. Although we find much better agreement between predictions and data, as shown for the thrust distribution in Fig. 2 (left) and for the distribution of heavy jet mass $\rho$ (= hemisphere invariant mass normalized by the total visible energy in the event) in Fig. 2 (right), the values of the extracted parameters (as shown in the plots) differ. Also, a strong anti-correlation was observed between the perturbative and non-perturbative parameters.

Another event shape variable, the energy-energy correlation [23], was studied at high precision recently in Ref. [24] combining the fixed-order prediction at NNLO [6] and the resummed one at NNLL [25]. This variable is computed as the energy-weighted cross section defined in terms of the angle $\theta_{i j}$ between the hadronic tracks of two particles $i$ and $j$ in an event,

$$
\frac{\mathrm{d} \Sigma(\chi)}{\mathrm{d} \chi}=\sum_{i, j} \int \frac{E_{i} E_{j}}{Q^{2}} \mathrm{~d} \sigma_{e^{+} e^{-} \rightarrow i j+X} \delta\left(\cos \chi+\cos \theta_{i j}\right),
$$

where $Q^{2}$ is the squared center-of-mass energy, $E_{i}$ and $E_{j}$ are the particle energies ${ }^{2}$. For this

\footnotetext{
${ }^{2}$ Setting $\cos \chi=-\cos \theta_{i j}$ using the $\delta$ distribution is a convention.
} 
observable the non-perturbative (NP) correction depends on two parameters $a_{1}$ and $a_{2}$ [26]. A fit of the NNLO+NNLL+NP prediction to OPAL and SLD data resulted in $\alpha_{\mathrm{s}}\left(M_{Z}\right)=0.121_{-0.003}^{+0.001}$ [24], but showed again very strong anti-correlations among $\alpha_{\mathrm{S}}\left(M_{Z}\right), a_{1}$ and $a_{2}$. Thus, we may conclude that the analytic models for hadronization are not sufficient to provide a precise and robust simultaneous estimation of the strong coupling and the non-perturbative parameters, which also questions the utility of some of the $\alpha_{\mathrm{s}}\left(M_{Z}\right)$ results quoted in the PDG [1].

As mentioned, one can also estimate the effects of hadronization on the distributions by using modern Monte Carlo models [27, 28]. As these are not based on first principles, the correct estimation of the hadronization uncertainty is ambiguous. Accepting the motto that "large uncertainty in small quantity is small uncertainty" we suggest the strategy of identifying observables for which the hadronization corrections are small. An example for an observable with small hadronization corrections is the jet cone energy fraction [29], for which also the NNLO corrections are very small except near the edges of the phase space [6]. Of course, small hadronization uncertainty does not immediately imply precise extraction of $\alpha_{\mathrm{s}}\left(M_{Z}\right)$, yet with large hadronization corrections we cannot expect precise $\alpha_{\mathrm{s}}\left(M_{Z}\right)$ extraction. To see this explicitly, we recall the results of two recent results of $\alpha_{\mathrm{s}}\left(M_{Z}\right)$ determination based on hadronic final states in lepton collisions. The first one is the energy-energy correlation mentioned above [19], which yielded

$$
\alpha_{\mathrm{s}}\left(M_{Z}\right)=0.11750 \pm 0.00018 \text { (exp.) } \pm 0.00102 \text { (hadr.) } \pm 0.00257 \text { (ren.) } \pm 0.00078 \text { (res.) }
$$

with a combined uncertainty \pm 0.00287 , i.e. about $2.5 \%$, dominated by the truncation of the perturbation series. This can be understood easily by the large radiative corrections to this distribution [6, 24], as shown in Fig. 3. Although significant progress has been achieved in the resummation of large logarithmic contributions for this observable [30, 31], the large radiative corrections may prevent a precise extraction of the strong coupling based on the energy-energy correlation.

Another recent determination of $\alpha_{\mathrm{s}}\left(M_{Z}\right)$ from jet rates [20] showed similar hadronization corrections, but smaller perturbative corrections, resulting in a reduced uncertainty:

$$
\alpha_{\mathrm{s}}\left(M_{Z}\right)=0.11881 \pm 0.00063 \text { (exp.) } \pm 0.00101 \text { (hadr.) } \pm 0.00045 \text { (ren.) } \pm 0.00034 \text { (res.) }
$$

This second extraction, based on $\mathrm{O}\left(\alpha_{\mathrm{s}}\left(M_{Z}\right)^{3}\right)$ predictions at fixed order and resummation of large logarithmic contributions at NNLL accuracy [32] for the two-jet rate, has a combined uncertainty \pm 0.00131 , i.e. only slightly above $1 \%$, being competitive with the lattice determination.

In this contribution we argued that precise determination of the strong coupling from hadronic final states in electron-positron annihilation requires (i) a careful selection of observables with as small radiative and hadronization corrections as possible, (ii) methods to estimate and reduce the effects of hadronization on the distributions, and (iii) estimation of the hadronization corrections with modern Monte Carlo tools. With these considerations taken into account the measurement of the strong coupling based on data to be collected at future lepton colliders promises an experimental determination of this fundamental parameter of the standard model with an uncertainty well below $1 \%$.

\section{References}

[1] Particle Data Group Collaboration, M. Tanabashi et. al., Review of Particle Physics, Phys. Rev. D98 (2018), no. 3030001 . 


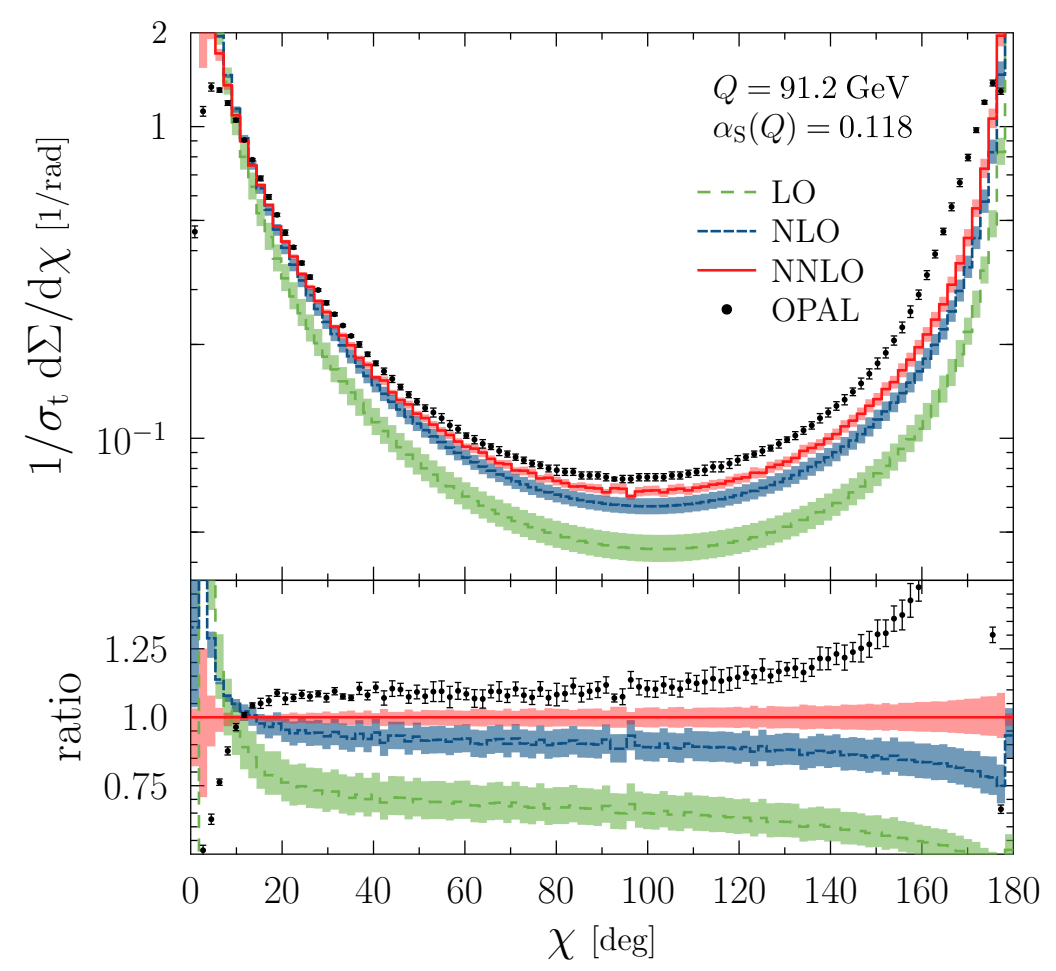

Figure 3: Energy-energy correlations at the first three orders in perturbation theory normalized to the total hadronic cross section and compared to OPAL data. The bands represent the variation of the renormalization scale as in Fig. 1.

[2] ALPHA Collaboration, M. Bruno, M. Dalla Brida, P. Fritzsch, T. Korzec, A. Ramos, S. Schaefer, H. Simma, S. Sint and R. Sommer, QCD Coupling from a Nonperturbative Determination of the Three-Flavor $\Lambda$ Parameter, Phys. Rev. Lett. 119 (2017), no. 10102001 [1706. 03821 ].

[3] D. d'Enterria, $\alpha_{s}$ status and perspectives (2018), in 26th International Workshop on Deep Inelastic Scattering and Related Subjects (DIS 2018) Port Island, Kobe, Japan, April 16-20, 2018, 2018. 1806.06156.

[4] A. Gehrmann-De Ridder, T. Gehrmann, E. W. N. Glover and G. Heinrich, NNLO corrections to event shapes in e+ e- annihilation, JHEP 12 (2007) 094 [0711.4711].

[5] S. Weinzierl, Event shapes and jet rates in electron-positron annihilation at NNLO, JHEP 06 (2009) $041[0904.1077]$.

[6] V. Del Duca, C. Duhr, A. Kardos, G. Somogyi, Z. Szőr, Z. Trócsányi and Z. Tulipánt, Jet production in the CoLoRFulNNLO method: event shapes in electron-positron collisions, Phys. Rev. D94 (2016), no. 7074019 [1606.03453].

[7] T. Becher and M. D. Schwartz, A precise determination of $\alpha_{s}$ from LEP thrust data using effective field theory, JHEP 07 (2008) 034 [0 803.0342 ].

[8] R. Abbate, M. Fickinger, A. H. Hoang, V. Mateu and I. W. Stewart, Thrust at $N^{3} L L$ with power corrections and a precision global fit for $\alpha_{s}\left(m_{Z}\right)$, Phys. Rev. D83 (2011) 074021 [1006. 3080 ]. 
[9] A. H. Hoang, D. W. Kolodrubetz, V. Mateu and I. W. Stewart, C-parameter distribution at $N^{3} L L$ including power corrections, Phys. Rev. D91 (2015), no. 9094017 [1411.6633].

[10] S. Brandt, C. Peyrou, R. Sosnowski and A. Wroblewski, The principal axis of jets. An attempt to analyze high-energy collisions as two-body processes, Phys. Lett. 12 (1964) 57-61.

[11] E. Farhi, A QCD test for jets, Phys. Rev. Lett. 39 (1977) 1587-1588.

[12] A. Kardos, G. Somogyi and Z. Trócsányi, Jet cross sections with CoLoRFulNNLO, PoS LL2016 (2016) 021.

[13] G. Somogyi, Z. Trócsányi and V. Del Duca, A subtraction scheme for computing QCD jet cross sections at NNLO: regularization of doubly-real emissions, JHEP 01 (2007) 070 [hep-ph/0609042].

[14] G. Somogyi and Z. Trócsányi, A subtraction scheme for computing QCD jet cross sections at NNLO: regularization of real-virtual emission, JHEP 01 (2007) 052 [hep-ph/ 0609043 ].

[15] A. Gehrmann-De Ridder, T. Gehrmann, E. Glover, and G. Heinrich, "EERAD3: event shapes and jet rates in electron-positron annihilation at order $\alpha_{s}^{3}$," Comput.Phys. Commun. 185 (2014) 3331, arXiv:1402.4140 [hep-ph].

[16] S. Catani, Y. L. Dokshitzer, M. Olsson, G. Turnock and B. R. Webber, New clustering algorithm for multi-jet cross-sections in e+ e- annihilation, Phys. Lett. B 269, 432 (1991).

[17] Y. L. Dokshitzer and B. R. Webber, Calculation of power corrections to hadronic event shapes, Phys. Lett. B 352, 451 (1995) [hep-ph/9504219].

[18] T. Gehrmann, G. Luisoni and P. F. Monni, Power corrections in the dispersive model for a determination of the strong coupling constant from the thrust distribution, Eur. Phys. J. C73 (2013), no. 12265 [1210.6945].

[19] A. Kardos, S. Kluth, G. Somogyi, Z. Tulipánt and A. Verbytskyi, Precise determination of $\alpha_{S}\left(M_{Z}\right)$ from a global fit of energyâĂŞenergy correlation to NNLO+NNLL predictions, Eur. Phys. J. C78 (2018), no. 6498 [1804.09146].

[20] A. Verbytskyi, A. Banfi, A. Kardos, P. F. Monni, S. Kluth, G. Somogyi, Z. Szőr, Z. Trócsányi, Z. Tulipánt and G. Zanderighi, High precision determination of $\alpha_{s}$ from a global fit of jet rates, JHEP 1908, 129 (2019) [1902.08158].

[21] G. P. Salam and D. Wicke, Hadron masses and power corrections to event shapes, JHEP 05 (2001) 061 [hep-ph/0102343].

[22] Y.-T. Chien and M. D. Schwartz, Resummation of heavy jet mass and comparison to LEP data, JHEP 08 (2010) 058 [1 005.1644$].$

[23] C. L. Basham, L. S. Brown, S. D. Ellis and S. T. Love, Energy correlations in electron-positron annihilation: testing QCD, Phys. Rev. Lett. 41 (1978) 1585.

[24] Z. Tulipánt, A. Kardos and G. Somogyi, Energy-energy correlation in electron-positron annihilation at NNLL + NNLO accuracy, Eur. Phys. J. C77 (2017), no. 11749 [1708. 04093 ].

[25] D. de Florian and M. Grazzini, The back-to-back region in e+e-energy-energy correlation, Nucl. Phys. B704 (2005) 387-403 [hep-ph / 0407241$].$

[26] Y. L. Dokshitzer, G. Marchesini and B. R. Webber, Nonperturbative effects in the energy energy correlation, JHEP 9907, 012 (1999) [hep-ph/9905339]. 
[27] J. Bellm et al., Herwig 7.0/Herwig++ 3.0 release note, Eur. Phys. J. C 76, no. 4, 196 (2016) [arXiv:1512.01178 [hep-ph]].

[28] T. Gleisberg, S. Hoeche, F. Krauss, M. Schonherr, S. Schumann, F. Siegert and J. Winter, Event generation with SHERPA 1.1, JHEP 0902, 007 (2009) [arXiv:0811.4622 [hep-ph]].

[29] Y. Ohnishi and H. Masuda, The jet cone energy fraction in e+e-annihilation, SLAC-PUB-6560.

[30] I. Moult and H. X. Zhu, Simplicity from recoil: the three-loop soft function and factorization for the energy-energy correlation, JHEP 1808, 160 (2018) [1801.02627].

[31] L. J. Dixon, I. Moult and H. X. Zhu, Collinear limit of the energy-energy correlator, Phys. Rev. D 100, no. 1, 014009 (2019) [1905.01310].

[32] A. Banfi, H. McAslan, P. F. Monni and G. Zanderighi, The two-jet rate in $e^{+} e^{-}$at next-to-next-to-leading-logarithmic order, Phys. Rev. Lett. 117 (2016), no. 17172001 [1607.03111]. 